\title{
Future of Statins in Sepsis: A Review on Its Safety and Efficacy
}

\author{
Sara M. Eladawy ${ }^{* a}$, Naglaa S. Bazan ${ }^{\mathrm{b}}$ \\ ${ }^{a}$ Clinical Pharmacy Department, Faculty of Pharmacy, Ain Shams University, Cairo 11566, Egypt \\ ${ }^{\mathrm{b}}$ Critical Care Medicine Department, Cairo University Hospitals, Cairo 11562, Egypt
}

\begin{abstract}
Sepsis is a life-threatening organ dysfunction caused by a dysregulated host response to infection. Statins [Hydroxymethylglutaryl-CoA reductase inhibitors] not only lower cholesterol levels but also have been proposed as adjunctive therapy in sepsis due to their pleiotropic effects. They act on several stages in sepsis: the generation of proinflammatory cytokines, modulation of leukocyte and monocyte functions, and reduction of oxidative stress as well as improvement in endothelial function and platelet activity. However, it has been argued if the observed beneficial effect of statins in sepsis is related to preadmission or post-admission use of statins. Also, the positive impact of statins on the clinical outcome of patients with sepsis has shown conflicting results. Accordingly, this review will discuss recent evidence regarding the use of statins in sepsis. Also, adequate use of statins based on the right drug, at the right time, at the right dose and in the right population will be discussed. The information in this review shows that the effect of statins is a drug, not a class effect, with the most effective drug in sepsis being simvastatin. Besides, it highlights the importance of proper timing and dosing of statins to manifest their antibacterial and pleiotropic effects. Finally, the effect of statins in sepsis is restricted to early phases of sepsis or sepsis prevention, not sepsis complicated with organ dysfunction or septic shock. However, more in vivo and clinical trials are required to determine the final decision about statin use in sepsis.
\end{abstract}

Keywords: statins, sepsis; preadmission; postadmission; pleitropic effects; antibacterial effects.

*Correspondence | Sara M. Eladawy; Clinical Pharmacy Department, Faculty of Pharmacy, Ain Shams University, Cairo 11566, Egypt. Email: sara.amin8445@yahoo.com

Citation | Eladawy SM, Bazan NS, 2020. Future of statins in sepsis: a review on its safety and efficacy. Arch Pharm Sci ASU 4(1): 20-35 DOI: 10.21608 /APS.2020.2001.1022

Print ISSN: 2356-8380. Online ISSN: 2356-8399.

Received 21 February 2020. Accepted 29 March 2020.

Copyright: ${ }^{{ }^{2}} 2020$ Eladawy et al. This is an open-access article licensed under a Creative Commons Attribution 4.0 International License (CC BY 4.0), which permits unrestricted use, distribution, and reproduction in any medium, provided the original author(s) and source are credited.

Published by: Ain Shams University, Faculty of Pharmacy

\section{INTRODUCTION}

Statins

[Hydroxymethylglutaryl-CoA reductase inhibitors] are the major and most effective therapeutic classes to lower cholesterol levels. However, due to their pleiotropic effects, statins have been proposed for the treatment of other conditions. The pleiotropic effects of statins are mainly related to their anti-inflammatory, anti-proliferative, antioxidant, neuroprotective, immunomodulatory, anti-diabetic and antithrombotic effects. Also, improving endothelial dysfunction and attenuating vascular remodeling, inhibition of Rho and its downstream target, Rho-associated coiled-coil-containing protein kinase [ROCK], their agonistic action on peroxisome proliferator-activated receptors [PPARs] and their anti-bacterial effect are among the important pleiotropic effects of statins $[1,2]$, Fig. 1. Based on those mechanisms, statins have been proposed to have a beneficial effect on sepsis. 


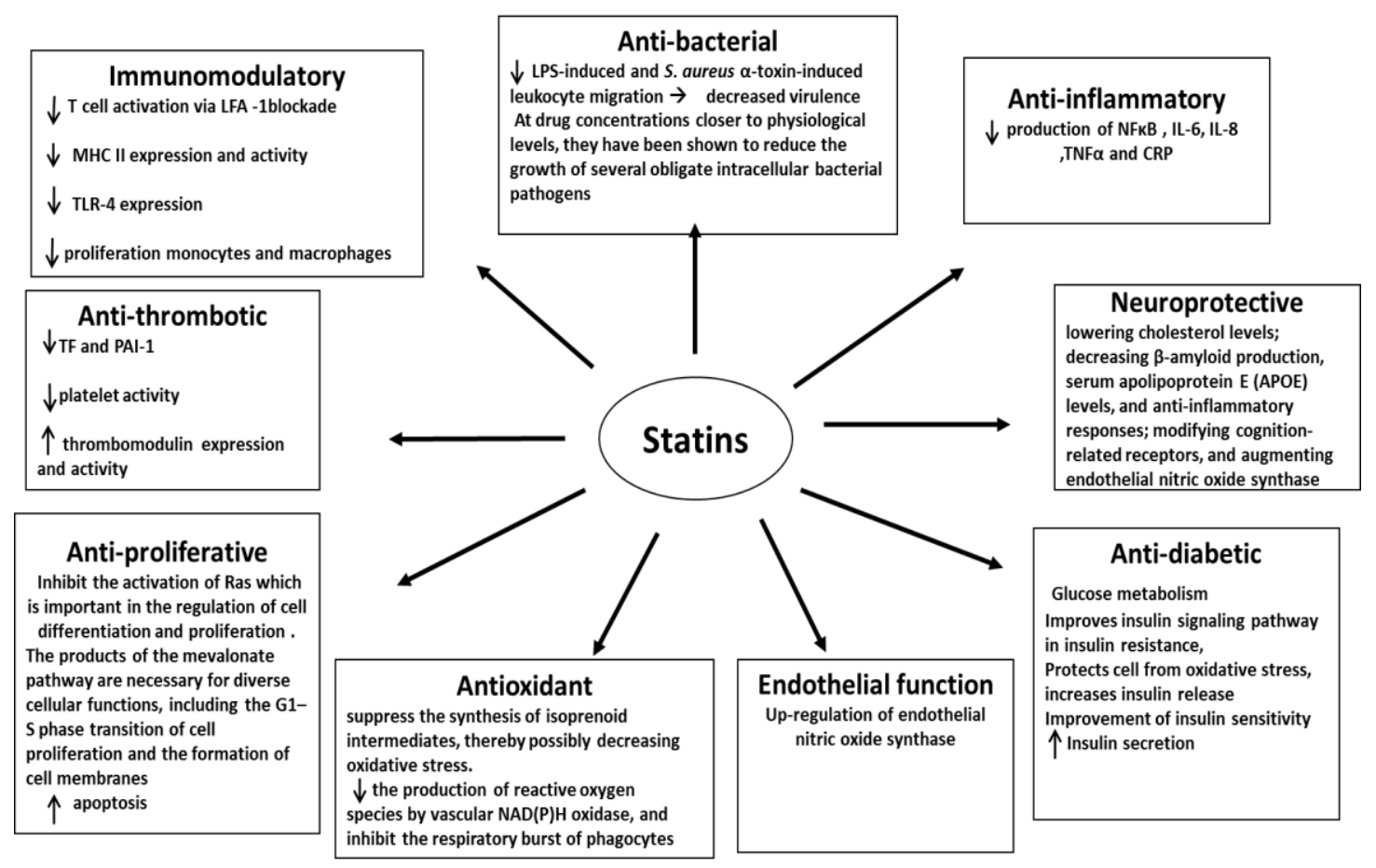

Fig 1. Statins Possible mechanisms and Effects in sepsis

Note NFאB, nuclear factor kappa-light-chain-enhancer of activated B cells; IL, interleukins; TNF $\alpha$, Tumor necrosis factor; CRP, C-reactive protein; NADPH, oxidase nicotinamide adenine dinucleotide Phosphate-oxidase; TF, tissue factor; PAI-1, plasminogen activator inhibitor-1; LFA -1, Leukocyte function-associated antigen; MHC II, Major histocompatibility class II; TLR-4, Toll-like receptor 4; LPS, Lipopolysaccharides.

Sepsis is a life-threatening organ dysfunction caused by a dysregulated host response to infection $[3,4]$. Hence, treatments that can reduce the impaired immune reaction in sepsis have been the focus of many studies in the last decade [5]. Accordingly, statins have been proposed as adjunctive therapy in sepsis. Observational studies have shown that outcome of sepsis was improved in patients who were on statins before admission to the hospital [6, 7]. On the other hand, randomized controlled trials [RCT] did not detect any positive impact when using statins in patients with sepsis postadmission [5]. In the current review, this contradiction will be argued at multiple levels.

\subsection{THE HEALTHY USER EFFECT}

The healthy user effect is a term used to describe patients on statins who tend to have a constellation of healthier attitudes and hence better outcomes in the majority of systemic diseases [5]. There is a debate relating to whether or not the protecting impact of preadmission use of statins was only owing to the healthy user effect. Possible healthy-user bias and ways to regulate it was discussed by Lee et al. [7] First, depending on predefined criteria for the serum lipid profile of the patients, reimbursement of statins in Taiwan is without co-payment. Second, they used validated codes for geographic areas which are highly related to the degree of urbanization to control socioeconomic status. Additionally, they controlled the insurance premium categories as a proxy for the private economic position. Moreover, proxies for several lifestyle factors were controlled. The main 
proxies were morbid obesity or alcohol-related disorders. Besides, to further ensure that they controlled healthy user bias, they decided to compare the propensity score [PS] matched nonusers to the amount of dental scaling for statin users. No significant change was shown in the latter comparison [7].

\subsection{SYSTEMATIC REVIEW CRITIC}

A systematic review and meta-analysis by Pertzov et al. concluded that using statins in the treatment of adult patients with sepsis is not recommended [8]. However, this was criticized by Zhou and Tang in 2019 who mentioned that this meta-analysis included a limited number of studies that can result in random errors and false results such as false-negative errors [type II errors]. To prove their point, they conducted a trial sequential analysis [TSA] to help in dealing with random errors and in calculating the optimal information size. The results of TSA showed that 12151 patients had optimal information, which is much more than the real number of 2562 patients [13 studies in total]. The general boundary line or any adjusted boundary line was not crossed by the $\mathrm{z}$ curve to favor the intervention group or control group proposing that future research is still required [9, 10]. Also, the latter study mentioned that Pertzov et al. criteria were incorrect where they incorporated studies of septic patients, as well as, those with infection and on mechanical ventilation [11-15]. They highlighted the importance of differentiating between the definition of sepsis as a composite of systemic inflammatory response and infection and the definition of infection alone. This definition was based on the 2001 Society of Critical Care Medicine [SCCM] / The European Society of Intensive Care Medicine [ESICM], the American College of Chest Physicians [ACCP], the American Thoracic Society [ATS], and the Surgical Infection Society [SIS] International sepsis definitions conference, which was adopted by Pertzov et al. $[\mathbf{8 , 9}$ ]. Also, they mentioned that the study by Shao 2016 had no available data on mortality and hence should have been excluded [16]. Accordingly, including publications of nonseptic patients plus the bounded number of studies comprised might undervalue the actual impact of statins on septic patients and make them appear ineffective.

\subsection{STATINS ADMINISTRATION ASPECTS}

Before closing the issue of using statins in sepsis, it would be reasonable to address some important aspects, including the use of the right drug, at the right time, in the right dose and right population. Those aspects will be discussed below:

\subsubsection{RIGHT DRUG (DRUG EFFECT NOT CLASS EFFECT)}

In 2016, new proof proposed that statins can change the bacterial virulence and have direct antibacterial effects [2]. Statins were suggested as an adjunctive host-directed therapy which is a cheap and useful substitute for the management of diseases produced by intracellular pathogens. The proposed mechanism is enhancing host defense mechanisms and inhibiting pathological inflammation and therefore decreasing mortality caused by infectious diseases in humans. The intermediates of the host mevalonate pathway are inhibited by statins at the cellular level, so undermining the survival of pathogens and their immune evasion strategies [17]. Those antibacterial or anti-virulence effects are not directly related to their capacity to reduce lipids but may be statin-specific [2]. For instance, although rosuvastatin lowers lipids to a greater extent than simvastatin, it was accompanied with less antibacterial effects than simvastatin [18] Moreover, the study by Lee et al. in 2018 evaluated mortality and statin prescription data from the Taiwan National Health Insurance 
Database from 2000-2011. The authors reviewed mortality data at 30- and 90-days in 52,737 patients diagnosed with sepsis. The statins used were atorvastatin, simvastatin, and rosuvastatin. All patients in the statin group included were on statins for at least 30 days before sepsis onset. Also, patients on multiple kinds of statins were excluded. There were no significant differences in comorbidities or other medications taken among the three statin groups or the non-users. The specific effect of statins on 30-day mortality revealed that simvastatin provided the greatest benefit on mortality [28\% risk reduction], followed by atorvastatin [22\% risk reduction]. The positive impact of statins was significant for the three statins at 90 days, although it was decreased. By applying 1:1:1 PS matching, a head-to-head comparison was conducted. The 30day mortality was significantly lower in simvastatin and atorvastatin compared to rosuvastatin. This positive impact of statins on mortality was not related to their potency in lowering lipids, but to statin's in vitro antibacterial activity, [5]. A review evaluating the antimicrobial effects of statins showed that simvastatin was the statin with the most potent antibacterial activity. The difference in chemical characteristics of simvastatin from pravastatin and atorvastatin has been suggested to be the reason behind the better antimicrobial activity of simvastatin compared to pravastatin and atorvastatin. Pravastatin and simvastatin are semi-synthetic forms that are derivatives of a metabolic product of Penicillium citrinum called lovastatin, while atorvastatin is the pure synthetic form. Also, pravastatin is hydrophilic, whereas, simvastatin and atorvastatin are lipophilic. Hence, those factors suggest that simvastatin may cross easily the cell membrane which leads to inhibition of the bacteria depending on the dose. On the other hand, atorvastatin had no antimicrobial activity despite its lipophilicity. This may be explained by the fact that atorvastatin is not obtained from a fungal metabolite [19].

It is worthy to mention that two studies were performed in animals to assess the role of statins in sepsis in the past 2 years. Braga et al. in 2018 concluded that using simvastatin as an adjuvant in the treatment of sepsis could be a good option to control the signs and changes the poor prognosis of sepsis and its associated conditions [20]. Those findings were reinforced by Qin et al. in 2019 which revealed that denovo simvastatin has a role in mitigating the symptoms of early sepsis [21]. Results of the latter two studies were supported in a human study published by our group in 2016. The study results showed that using $40 \mathrm{mg}$ simvastatin in intensive care unit patients with early sepsis resulted in a significant reduction in Intensive Care Unit [ICU] length of stay [ICU LOS] either in total patients or after repeated test on alive patients. Also, simvastatin arm reduced mortality in comparison with the standard control arm but failed to reach statistical significance [22]. Besides, a recent study showed that simvastatin protects the vascular endothelium in rats with sepsis. In the latter study, simvastatin significantly reduced blood serum pseudo hemophilia factor (vWF), thrombomodulin (TM), platelet-activating factor (PAF), antithrombin-III (AT-III) levels in sepsis thus it can protect vascular endothelial from damage and improve coagulation disorders in sepsis [23].

\subsubsection{TIMING}

An important point to appraise is the timing of using statins and whether their effect on sepsis is related to the timing of starting statins. The two groups suggested are either those who were already on statins or those who received denovo treatment. An observational study by Lee et al., 2017 found that using statins before admission showed a small and significant improvement in 
survival when compared with patients who did not receive statins before. This favorable impact on survival was more obvious in patients with lower severity. Thus, prophylaxis from sepsis using statins in high-risk patients like those who are immunocompromised or have a history of sepsis may be promising [7]. Also, $\mathrm{Hu}$ et al., 2019 examined the advantages of statins on the long term prognosis of patients who had survived sepsis. They included a total of 220,082 patients with the first episode of sepsis who had been hospitalized between 1999 and 2013. The results showed that 134,448 [61.09\%] of them were alive at discharge. The users of statins were defined as the alive patients who were on statins at a concentration of more than 30 cumulative Defined Daily Doses [cDDDs] during post-sepsis discharge. The study showed that statins may have the capability to reduce the long-term mortality of patients who survived after sepsis. Yet, more data is required to prove the results [24]. This effect was not proven in any RCT. The use of statins before hospital admission was mostly evaluated in observational studies, while RCTs assessed the usage of statins for the first time after hospital admission. An exception was an RCT performed by Kruger and colleagues who reported that patients who were already on statins for two weeks or more had a $23 \%$ lower 28-day mortality, while no effect was observed in those who used statins for the first time at the hospital. However, the results of the latter study were not generalized because of its small sample size [25]. Another important issue to discuss is whether certain treatment duration is needed for statins to manifest their anti-inflammatory properties. Plenge et al. conducted a crossover double-blind trial which showed that statin therapy of at least 14 days is needed to decrease C-reactive protein serum levels [26]. Also, a meta-analysis that included eight RCTs conducted by Chen et al. reported an opposite correlation between the time on statins before admission and the risk of occurrence of atrial fibrillation postoperatively. The latter analysis showed that patients who were on statins for $>90$ days duration had better survival than those who were on statins for 7-90 days duration [27]. Additionally, regarding RCTs, the majority were conducted on critically ill patients with severe sepsis or septic shock. However, it was proposed by Lee et al, 2017 that using only statins is not enough to positively change the outcome of severely ill patients. In their subgroup analysis, it was suggested that previous use of statin did not have favorable effects in severely ill patients who require ICU admission or mechanical ventilation [7]. Similarly, Chen et al., 2013 evaluated the impact of adequate empirical antibiotic therapy on bloodstream infections. They reported that adequate empirical antibiotic therapy in patients with high mortality in Emergency Department Sepsis (MEDs) scores did not display a considerable favorable effect while it was clearer in patients with a lower MEDS scores [28].

\subsubsection{RIGHT DOSE}

\subsubsection{PLEIOTROPIC EFFECTS}

Preclinical studies show that the threshold for an anti-inflammatory effect of statins surpasses that its effect in lowering lipids. The observational study by Van der Meij et al., 2013 offers obvious evidence for a dose-dependent but highly selective effect of statins on vascular inflammation [29]. Similarly, Ou et al. proved that high-potency statins and low potency statins were associated with a $20 \%$ and $11 \%$ reduced risk of 1-month mortality after sepsis, respectively. Those receiving at least $10 \mathrm{mg}$ rosuvastatin, at least $20 \mathrm{mg}$ atorvastatin, or at least $40 \mathrm{mg}$ simvastatin were defined as highpotency statin users, while those receiving all other statin treatments were defined as lowpotency statin users [30]. It is worth mentioning that low statin doses have proangiogenic effects [31], which may be fatal in severe sepsis [32]. 


\subsubsection{ANTIBACTERIAL EFFECTS}

Statins' effect on in vitro virulence of some pathogens is promising but is restrained by the need for high concentrations to achieve significant results [2]. The blood concentrations attained in patients undergoing statin therapy were found to be a thousand times less than concentrations associated with antimicrobial properties [33]. Nevertheless, this can be conquered by combining antibiotics with the use of sub-inhibitory concentrations of statins. Although promising, the evidence of using this combination of statins with antibiotics may be statin/pathogen-specific. Also, the risk of adverse effects due to drug-drug interactions should be considered [2].

Also, it has been shown that simvastatin causes bacterial inhibition in a dose-dependent manner [19]. Hence, the two major concerns of using simvastatin as anti-bacterial are its safety in higher doses and whether concentrations of antimicrobials found in vitro would be attainable in blood, bearing in mind its poor solubility [19]. Currently, a new simvastatin nanostructured lipid carrier's formulation [SV-NLCs] is under investigation that may enhance available in vivo concentration hence increase the efficacy.

\subsubsection{RIGHT POPULATION}

The three major types of sepsis are bacterial, viral, and fungal sepsis. While similar in symptoms, each has its mechanism of action. Additionally, the etiologies and immune mechanisms of these types differ enough that a distinct patient base can be identified for each one. General treatment, such as broad-spectrum antibiotics, without knowing the origin of sepsis may exacerbate symptoms and result in a significant rise in morbidity and mortality [34]. Moreover, many factors contribute to an individual's outcome in sepsis. This difference in outcome is mainly because sepsis originates from different causes, affects various groups of patients, with differing severity, and is greatly changed by the presence of comorbidities [35].

Lee et al. mentioned that although most RCTs evaluating the role of statins in sepsis were on severely ill patients in the ICU, the positive impact of statins did not occur in patients needing to be admitted to the ICU or patients with acute organ dysfunctions [5]. They accredited that to the fact that the potential benefits of statins are principally by decreasing inflammation via intracellular signaling, lowering catecholamine levels, or decreasing Toll-like receptor activation by pathogen-associated molecular patterns [PAMPs]. Also, at large doses, statins have antiangiogenic and antioxidant effects, which may avoid the progress of severe sepsis. However, high levels of substances like lipopolysaccharides and lipoteichoic acids [i.e catecholamines and PAMPs] lead to a primary pro-inflammatory response in early sepsis. Primary inflammation is concurrently associated with the initiation of anti-inflammatory feedback and in certain cases, the secondary inflammatory response may be produced by secondary infections. In case there is a progression in sepsis, vagal stimulation occurs because of the pathogenic bacteria to reduce catecholamines and halt the immune system of the host. After the onset of sepsis, additional pro-inflammatory factors arise slightly later after the start of sepsis which includes cachexia, protein catabolism, persistent inflammation and anti-inflammatory factors [defects in adaptive immunity] [36]. It is worth mentioning that role of statins in sepsis was underestimated when studies assessed their role in septic patients with and without ARDS This is evidenced by a recent study that showed that pretreatment with pravastatin in sepsis appears to prevent intestinal hypoxia through the improvement of the intestinal microvascular oxygenation. However, this effect is abolished by 
additional hypercapnia, signifying why pravastatin therapy in septic ARDS patients might not be beneficial [37].

\subsection{STATINS CONTROVERSIAL ISSUES}

A pragmatic definition of statin intolerance has been suggested by the Canadian Consensus Working Group 2016. They defined goal inhibiting statin intolerance as a clinical syndrome characterized by significant symptoms and/or biomarker abnormalities that prevent long term use of and adherence to indicated use of statins as documented by challenge/dechallenge/re-challenge, when appropriate, using at least two statins, including atorvastatin and rosuvastatin, that is not due to drug-drug interactions or untreated risk factors for intolerance [e.g. untreated hypothyroidism], and leading to failure to maintain therapeutic goals as defined by national guidelines. [38]. Nevertheless, true identification of statin toxicity or intolerance remains hindered by a lack of clear definitions and biomarkers [39]. Difficulties in the identification and diagnosis of statin intolerance, particularly concerning muscle symptoms, led to a controversy in its prevalence [40]. Observational studies propose it happens in $10 \%$ to $15 \%$ of patients, $[\mathbf{4 1}, \mathbf{4 2}]$ with clinic data reporting to reach $30 \%[\mathbf{4 3}, \mathbf{4 4}]$. On the other hand, the incidence is suggested to be $1.5 \%$ to $5 \%$ of patients in RCTs, although this is probably an underestimation because patients with a history of statin intolerance either before randomization or during the run-in period are usually excluded $[38,41,45,46]$.

\subsubsection{STATIN-ASSOCIATED MUSCLE SYMPTOMS [SAMS]}

Statin-associated muscle symptoms [SAMS] are the most prevalent and important adverse event of statins where $72 \%$ of all statin adverse events being muscle-related [47]. These can present as myalgia, myopathy, myositis with elevated CK [creatinine kinase], or rhabdomyolysis, with some people reporting additional joint and abdominal pain [48]. Other skeletal-related side effects include tendinopathies and tendon disorders, as well as arthralgias, although these are rarely evaluated in large RCTs [49]. Several groups have worked to provide a unified definition and diagnostic approach for SAMS [50, 51]. Risk factors to SAMS are either drug-related risk factors [Lipophilic and high dose statin] or patientrelated [history of myopathy with another lipidlowering drug, unexplained cramps, high creatinine kinase, low body mass index, untreated hypothyroidism, old age, and female sex]. Other risk factors include vitamin $\mathrm{D}$ deficiency and calcium disorders [39]. Retrospective, crosssectional, and meta-analysis reveal an association between low vitamin D levels and myalgia in patients on statin therapy $[52,53]$. A recent secondary analysis trial has shown that monthly vitamin D supplementation results in improved adherence to statin medication in older adults on long-term statin therapy [54].

\subsubsection{THE LIVER INJURY}

On starting any statin, about $0.5-2.0 \%$ of patients show a mild elevation in liver transaminases within 3 months of its initiation. However, this is unlikely to be clinically relevant [55, 56]. Ironically, based on some studies, statins have been shown to have clinical benefits in patients with liver disease, including nonalcoholic fatty liver disease [NAFLD], cholestatic liver diseases, and cirrhosis. Statins have demonstrated improvement in functional alterations and liver histology in patients with NAFLD, while also decreasing the risk of cardiovascular events [57]. Additionally, a reduction in portal hypertension in patients with cirrhosis, and reduction in the risk of decompensation and death in this population have been shown with statin use [58]. Hence, 
although, statin use was not recommended for many years in patients with elevated liver enzymes, there is promising evidence in their possible positive effects in patients with liver disease [59].

\subsubsection{NEW-ONSET TYPE 2 DIABETES MELLITUS [DM]}

Treatment with statins has led to a moderate rise in the risk of newly diagnosed DM of about one case per 1000 patients per year of exposure and has been shown to prevent five new coronary vascular diseases [CVD] events in genetic studies and RCTs. People with metabolic syndrome or prediabetes have a higher risk of this adverse effect. However, patients should be calmed that the beneficial effects of statins in protecting from CVD offset the potential risk from the increase in glucose level in plasma, especially in individuals with increased HbA1c [60].

\subsubsection{NEUROLOGICAL NEUROCOGNITIVE CONDITIONS}

AND

Epidemiological studies have shown that high cholesterol levels are associated with increased risk of Alzheimer's disease [61, 62], leading to the assumption that improving vascular function with statin therapy might be helpful in the context of many pathologies causing dementia [63]. On the contrary, statins have been proposed to be harmful to cognitive function due to their cholesterol-lowering effect [64]. Nevertheless, the brain-blood barrier and the reality that the brain is largely self-sufficient regarding endogenous cholesterol synthesis justifies the fact that statins have a direct effect on the brain [65].

\subsubsection{RENAL TOXICITY}

There is a disagreement concerning the effects of statins on renal function. All statins are metabolized by the liver and minimally cleared by the kidney except for hydrophilic statins [pravastatin and rosuvastatin]. Also, mild transient proteinuria sometimes occurs when using high doses of statin, but this does not lead to renal impairment [60]. Of interest, the use of atorvastatin has been proposed to reduce inflammation and improve kidney function after transplantation [66].

\subsubsection{CORONARY CALCIFICATION}

ARTERY

Statins act as mitochondrial toxins that damage the function of the muscle in the heart and blood vessels through the consumption of coenzyme Q10 and B heme A and thus adenosine triphosphate [ATP] generation. Besides, they block the synthesis of vitamin $\mathrm{K}_{2}$, the cofactor for matrix Gla-protein activation, which leads to protecting arteries from calcification. Moreover, impairment of selenoprotein biosynthesis which leads to congestive heart failure and reminiscent of the dilated cardiomyopathies seen with selenium deficiency has been related to statins. Nevertheless, information regarding the exact statin that leads to coronary artery calcification is not well defined [67].

\subsubsection{CANCER}

The carcinogenicity of statins has been related to their lipid-lowering effects [68]. However, also they have been suggested to prevent colorectal cancer [69]. Ravnskov U et al. criticized the latter study by discussing that cancer has been associated with low cholesterol levels measured 10 to 30 years before diagnosis in nine studies and that evidence associating statins with cancer is much stronger. $[68,70]$ Also, several studies in humans and animals have associated statins with carcinogenicity [70]. Currently, it is challenging to prove either assumption.

\subsubsection{MICROBIOME-MEDIATED EFFECT}

Recent studies have demonstrated a role for statins in modulating microbiome composition, 
with statin therapy resulting in profound remodeling of the gut microbiota, hepatic gene deregulation, changes in bile acid pool size and composition, as well as metabolic alterations in mice through a pregnane $X$ receptor-dependent mechanism [71]. Simvastatin has been shown to influence gut-derived metabolites which may impact response to the drug, as well as the development of adverse events [72].

\subsection{DRUG-DRUG INTERACTIONS}

The most common drugs associated with statin drug interactions are glucocorticoids, antipsychotics, human immunodeficiency virus protease inhibitors, azole antifungal agents, immunosuppressive drugs, macrolides, calcium channel blockers, and lipid-modifying drugs like gemfibrozil. Additionally, interactions can also occur with alcohol, opioid, and cocaine abuse [73]. Despite that, the variability in statin disposition in terms of metabolizing enzymes and transporters, as well as inter-individual differences in the activity of Cytochrome [CYP450] enzymes and transport proteins, makes statin drug-drug interactions hard to anticipate [74].

\subsection{CHALLENGES}

Statins are tempting candidates for use in several therapeutic indications due to their pleiotropic effects, safety record, efficacy, and affordability. However, there are still several important questions that need to be answered. The first issue to be addressed is the direct antibacterial activity of statins. They have the qualities of antimicrobial resistance [AMR] "breakers", however, their role in synergizing with antibiotics, and capability to prompt the host immune system, is limited. This is because their ongoing wide use for cardiovascular protection might lead to selective pressures for resistance, paradoxically leading statins to be AMR "makers". The study by Ko et al, addressing that matter concluded that among statins, simvastatin may be the best statin tailored for use as a novel adjuvant antibiotic. At present, the evidence is still in favor of considering statins as AMR breakers, but their probability of being AMR makers cannot be ruled out [36]. Besides, there is a necessity for a complete understanding of statins' chemical properties and the exact mechanism of action. Besides, caution to significant drug-drug interaction especially with drugs that are metabolized by the same cytochromes as statins, which were primarily examined in the form of small- scale trials [17]. Finally, a new formulation is under investigation to enhance the simvastatin very low oral bioavailability $[<5 \%]$ and lengthen its lipidlowering effect. A nanostructured lipid carrier [NLCs] of the antihyperlipidemic drug simvastatin was developed to enhance its bioavailability. As compared to the simvastatin suspension, oral administration of a single dose of simvastatin-NLC resulted in a 4-fold increase in bioavailability. Therefore, NLCs might offer efficient and hopeful drug delivery systems to increase simvastatin oral bioavailability [75] Moreover, a recent study showed that endothelial barrier function can be restored by using simvastatin-NLCs preparations through restoring endothelial cell migration, which is involved in the regulation of the PDGFR $\beta / P I 3 K / A k t / I Q G A P 1$ pathway and platelet-derived growth factor PDGF-BB secretion. As an applicable formulation for restoring endothelial dysfunction, simvastatinNLCs may be more effective than simvastatin [76].

\section{CONCLUSION}

Effect of statins is a drug, not a class effect, with the most effective drug in sepsis being simvastatin. Besides, statins require proper time and dosing to manifest their antibacterial and pleiotropic effects. Finally, based on that review, 
current evidence suggests that the effect of statin in sepsis is restricted to early phases of sepsis or sepsis prevention, not sepsis complicated with organ dysfunction or septic shock. However, more in vivo and randomized clinical trials are required to determine the final decision about statin use in sepsis

\section{DECLARATIONS}

The authors have no relevant affiliations or financial involvement with any organization or entity with a financial interest in or financial conflict with the subject matter or materials discussed in the manuscript. This includes employment, consultancies, honoraria, stock ownership or options, expert testimony, grants or patents received or pending, or royalties. No writing assistance was utilized in the production of this manuscript.

\section{ETHICS APPROVAL AND CONSENT TO PARTICIPATE}

Not applicable.

\section{COMPETING INTERESTS}

The authors declare that there is no conflict of interest.

\section{FUNDING STATEMENT}

No funding was received.

\section{ABBREVIATIONS}

ROCK, Rho-associated coiled-coil-containing protein kinase; PPARs, Peroxisome proliferatoractivated receptors; RCT, Randomized controlled trials; PS, A propensity score; SCCM, Society of Critical Care Medicine; ESICM, The European Society of Intensive Care Medicine; ACCP, The American College of Chest Physicians; ATS, The American Thoracic Society; SIS, Surgical Infection Society; TSA, Trial sequential analysis; ICU LOS, Intensive Care Unit length of stay; vWF, Serum pseudo hemophilia factor; TM, Thrombomodulin; PAF, Platelet-activating factor; AT-III, Antithrombin-III cDDDs, Cumulative Defined Daily Doses; MEDS, Mortality in Emergency Department Sepsis; SVNLCs, Simvastatin nanostructured lipid carriers; PAMPs, Pathogen associated molecular patterns; SAMS, Statin-associated muscle symptoms; CK, Creatinine kinase; NAFLD, Non-alcoholic fatty liver disease

HVPG, Hepatic venous pressure gradient; DM, Diabetes Mellitus; CVD, Coronary vascular diseases; ATP, Adenosine triphosphate; CARE, Cholesterol and Recurrent Events; PROSPER, Prospective Study of Pravastatin in the Elderly at Risk; SEAS, Simvastatin and Ezetimibe in Aortic Stenosis; CYP450, Cytochrome P450; AMR, Antimicrobial resistance; PDGF, Platelet-derived growth factor; ARDS, acute respiratory distress syndrome

\section{REFERENCES}

1. Bedi O, Dhawan V, Sharma P. L and Kumar P. Pleiotropic effects of statins: new therapeutic targets in drug design.NaunynSchmiedeberg's Arch Pharmacol 2016; 389:695-712.DOI 10.1007/s00210-016-12524

2. Hennessy E, Adams C, Reen FJ, O'Gara F. Is there potential for repurposing statins as novel antimicrobials? Antimicrob Agents Chemother. 2016; 60:5111-21. doi: 10.1128/AAC.00192-16. Print 2016 Sep.

3. Singer M, Deutschman CS, Seymour C W, Shankar-Hari M, Annane D, Bauer M, et al. The Third International Consensus Definitions for Sepsis and Septic Shock [Sepsis-3]. JAMA. 2016; 315:801-10 doi:10.1001/jama.2016.0287

4. Rhodes A, Evans LE, Alhazzani W, Levy MM, Antonelli M, Ferrer R, et al. Surviving Sepsis Campaign: International Guidelines for Management of Sepsis and Septic Shock: 2016. Intensive Care Med 2017; 43:304-77. 
DOI: $10.1007 / \mathrm{s} 00134-017-4683-6$

5. Lee CC, Lee M G, Hsu TC, Porta L, Chang $\mathrm{SS}$, Yo $\mathrm{CH}$, et al. A Population-Based Cohort Study on the Drug-Specific Effect of Statins on Sepsis Outcome. Chest 2018; 153:805-15. DOI: 10.1016/j.chest.2017.09.024

6. Al Harbi SA, Tamim HM, Arabi YM. Association between statin therapy and outcomes in critically ill patients: a nested cohort study. BMC Clin Pharmacol. 2011;11:12. doi: 10.1186/1472-6904-11-12.

7. Lee M. G, Lee C.C, Lai C.C, Hsu T.C, Porta $\mathrm{L}$, Lee $\mathrm{M}$, et al. Preadmission statin use improves the outcome of less severe sepsis patients - a population-based propensity score-matched cohort study. British Journal of Anaesthesia 2017; 119: 645-54. doi: 10.1093/bja/aex294

8. Pertzov B, Eliakim-Raz N, Atamna $\mathrm{H}$, Trestioreanu AZ, Yahav D, Leibovici L. Hydroxymethylglutaryl-CoA reductase inhibitors [statins] for the treatment of sepsis in adults - a systematic review and metaanalysis. Clin Microbiol Infect 2019; 25:28089. DOI: $10.1016 /$ j.cmi.2018.11.003

9. Zhou $X$ and Tang G, Re: 'Hydroxymethylglutaryl-CoA reductase inhibitors [statins] for the treatment of sepsis in adults' by Pertzov et al., Clinical Microbiology and Infection 2019, 25:1570-71 https://doi.org/10.1016/j.cmi.2019.05.005

10. Thorlund K, Engstrøm J, Wetterslev J, Brok J, Imberger G, Gluud C. User manual for trial sequential analysis [TSA]. 2017. Available from: http://www.ctu.dk/tsa/files/TSA_manual.pdf

11. Makris D, Manoulakas E, Komnos A, Papakrivou E, Tzovaras N, Hovas A, et al. Effect of pravastatin on the frequency of ventilator-associated pneumonia and intensive care unit mortality: open-label, randomized study. Crit Care Med 2011; 39:2440e6. doi: 10.1097/CCM.0b013e318225742c.

12. McAuley DF, Laffey JG, O'Kane CM, Perkins GD, Mullan B, Trinder TJ, et al. Simvastatin in the acute respiratory distress syndrome. N Engl J Med 2014; 371:1695e703. doi: 10.1056/NEJMoa1403285. Epub 2014 Sep 30.

13. Papazian L, Roch A, Charles PE, PenotRagon C, Perrin G, Roulier P, et al. Effect of statin therapy on mortality in patients with ventilator-associated pneumonia: a randomized clinical trial. JAMA 2013;310:1692e700. doi: 10.1001/jama.2013.280031.

14. Novack V, Eisinger M, Frenkel A, Terblanche M, Adhikari NK, Douvdevani A, et al. The effects of statin therapy on inflammatory cytokines in patients with bacterial infections: a randomized double-blind placebo-controlled clinical trial. Intensive Care Med 2009;35:1255e60

15. Versus D, Garcia-Vidal C, Simonetti AF, Dorca J, Llopis F, Mestre M, et al. The effect of simvastatin on inflammatory cytokines in community-acquired pneumonia: a randomized, double-blind, placebo-controlled trial. BMJ Open 2015; 5, e006251. doi: 10.1007/s00134-009-1429-0. Epub 2009 Feb 11.

16. Shao H, Wang C, Zhu W, Huang X, Guo $\mathrm{Z}$, Zhang $\mathrm{H}$, et al. Influence of simvastatin treatment on Toll-like receptor 4 in monocytes of peripheral blood in patients with sepsis and severe sepsis. Zhonghua wei zhong bing ji jiu yi xue 2016;28:159e63. doi: 10.3760/cma.j.issn.2095-4352.2016.02.014.

17. Parihar S.P , Guler R and Brombacher F. Statins: a viable candidate for host-directed 
therapy against infectious diseases. nature reviews immunology 2019; 19:105-17. doi: 10.1038/s41577-018-0094-3.

18. Masadeh M, Mhaidat N, Alzoubi K, AlAzzam S, Alnasser Z. Antibacterial activity of statins: a comparative study of atorvastatin, simvastatin, and rosuvastatin. Ann Clin Microbiol Antimicrob 2012; 11:13. doi: 10.1186/1476-0711-11-13.

19. Graziano TS, Cuzzullin $\quad$ MC, Franco GC, Schwartz-Filho HO, de Andrade ED, Groppo FC, et al. Statins and Antimicrobial Effects: Simvastatin as a Potential Drug against Staphylococcus aureus Biofilm. Plos one 2015; 10:e128098 . DOI: 10.1371/journal.pone.0128098

20. Braga Filho JAF, Abreu AG, Rios CEP, Trovão LO, Silva DLF, Cysne DN, et al. Prophylactic Treatment With Simvastatin Modulates the Immune Response and Increases Animal Survival Following Lethal Sepsis Infection. Front. Immunol 2018; 9:2137. doi: 10.3389/fimmu.2018.0213

21. Qin L, Xie X, Fang P, Lin J. Prophylactic simvastatin treatment modulates the immune response and increases the survival of mice following induction of lethal sepsis. Journal of International Medical Research 2019; 47:3850-9. doi: 10.1177/0300060519858508. Epub 2019 Jul 16.

22. El Hamamsy M, Amin S, Bazan N, Elgendy AM, Zaki MA. The clinical outcome of simvastatin plus standard therapy versus standard therapy alone in critically ill septic patients: Randomized controlled clinical trial. Eur J Clin Pharm 2016; 18: 296303. www.farmclin. com/seccion. asp? $\mathrm{Id}=8 \&$ art iculo $=1125$

23. Lyu XC, Cai GL, Xu QH, Hu CB, Yan ML, Zhang HH. Changes of serum pseudohemophilia factor, thrombomodulin, platelet-activating factor, antithrombin-III in sepsis rats and the intervention effect of simvastatin . Chinese Journal of Internal Medicine, 2020; 59 (1): 52-57. DOI: 10.3760 / cma.j.issn.0578-1426.2020.01.009

24. Hu S Y, Hsieh MS, Lin TC, et al. Statins improve the long-term prognosis in patients who have survived sepsis. A nationwide cohort study in Taiwan [STROBE complaint]. Medicine 2019; 98:17[e15253]. doi.org/10.1097/MD.0000000000015253

25. Kruger P, Bailey M, Bellomo R, Cooper DJ, Harward M, Higgins A, et al. A multicenter randomized trial of atorvastatin therapy in intensive care patients with severe sepsis. Am J Respir Crit Care Med 2013; 187: 743-50. doi: 10.1164/rccm.201209-1718OC.

26. Plenge JK, Hernandez TL, Weil KM, Poirier $\mathrm{P}$, Grunwald GK, Marcovina SM, et al. Simvastatin lowers C-Reactive protein within 14 days an effect independent of low-density lipoprotein cholesterol reduction. Circulation 2002; 106: 1447-52. DOI: 10.1161/01.cir.0000029743.68247.31

27. Chen WT, Krishnan GM, Sood N, Kluger J, Coleman CI. Effect of statins on atrial fibrillation after cardiac surgery: a durationand dose-response meta-analysis. $\mathrm{J}$ Thorac Cardiovasc Surg 2010; 140: 364-72. doi: 10.1016/j.jtcvs.2010.02.042. Epub 2010 Apr 9.

28. Chen HC, Lin WL, Lin CC, Hsieh WH, Hsieh $\mathrm{CH}, \mathrm{Wu} \mathrm{MH}$, et al. Outcome of inadequate empirical antibiotic therapy in emergency department patients with community-onset bloodstream infections. J Antimicrob Chemother 2013; 68: 947-53. doi: 10.1093/jac/dks475. Epub 2012 Dec 21.

29. Van der Meij E, Koning GG, Vriens PW, 
Peeters MF, Meijer CA, Kortekaas KE, et al. A Clinical Evaluation of Statin Pleiotropy: Statins Selectively and Dose- Dependently Reduce Vascular Inflammation. PLoS ONE 2013; $\quad$ 8: e53882. doi:10.1371/journal.pone.0053882

30. Ou SY, Chu H, Chao PW, Ou S.M, Lee Y.J, Kuo S.C, et al. Effect of the use of low and high potency statins and sepsis outcomes. Intensive Care Med 2014; 40:1509-17. DOI 10.1007/s00134-014-3418-1

31. Gazzerro P, Proto MC, Gangemi G, Malfitano AM, Ciaglia E, Pisanti S, et

al. Pharmacological actions of statins: a critical appraisal in the management of cancer. Pharmacological Reviews 2012; 64:102-146. doi: 10.1124/pr.111.004994. Epub 2011 Nov 21.

32. Vera S, Martinez R, Gormaz JG, Gajardo A, Galleguillos F, Rodrigo R. Novel relationships between oxidative stress and angiogenesis-related factors in sepsis: new biomarkers and therapies. Annals of Medicine 2015; 47:289-300. doi: 10.3109/07853890.2015.1029967. Epub 2015 May 21.

33. Bergman P, Linde C, Putsep K, Pohanka A, Normark S, Henriques-Normark B, et al. Studies on the antibacterial effects of statinsin vitro and in vivo. PloS one 2011; 6:e24394. doi: 10.1371/journal. pone.0024394

34. Dolin HH, Papadimos TJ, Chen X, and Pan ZK. Characterization of Pathogenic Sepsis Etiologies and Patient Profiles: A Novel Approach to Triage and Treatment. Microbiology Insights 2019; 12: 1-8. DOI: 10.1177/1178636118825081

35. Kruger PS and Terblanche M. Statins in patients with sepsis and ARDS: is it over? $\mathrm{N}$. Intensive Care Med 2017; 43:675-676. DOI 10.1007/s00134-016-4564-4
36. Ko HHT, Lareu RR, Dix BR, Hughes JD. Statins: antimicrobial resistance breakers or makers? PeerJ 2017; 5:e3952 https://doi.org/10.7717/peerj.3952

37. Schulz J, Vollmer C, Truse R, Bauer I, Beck C, Picker O, Herminghaus A. Effect of Pravastatin Pretreatment and Hypercapnia on Intestinal Microvascular Oxygenation and Blood Flow During Sepsis. Shock. 2020; 53(1):88-94. doi: 10.1097/SHK.0000000000001323

38. Mancini GB, Baker S, Bergeron J, Fitchett D, Frohlich J, Genest J, et al. Diagnosis, prevention, and management of statin adverse effects and intolerance: Canadian Consensus Working Group Update [2016]. Can J Cardiol 2016; 32:S35-S65. doi: 10.1016/j.cjca.2016.01.003

39. Ward NC, Watts GF, Eckel RH. Statin Toxicity Mechanistic Insights and Clinical Implications. Circ Res 2019; 124:328350.DOI:10.1161/CIRCRESAHA.118.312782

40. Saxon DR and Eckel RH. Statin intolerance: a literature review and management strategies. Prog Cardiovasc Dis. 2016; 59:153-164. doi: 10.1016/j.pcad.2016.07.009

41. Banach M, Rizzo M, Toth PP, Farnier M, Davidson MH, Al-Rasadi K, et al. Statin intolerance - an attempt at a unified definition. A position paper from an International Lipid Expert Panel. Arch Med Sci. 2015; 11:1-23. doi: 10.5114/aoms.2015.49807

42. Tobert JA and Newman CB. Statin tolerability: in defense of placebo-controlled trials. Eur J Prev Cardiol. 2016; 23:891-896. doi: $10.1177 / 2047487315602861$

43. Keen HI, Krishnarajah J, Bates TR, Watts GF. Statin myopathy: the fly in the ointment for the prevention of cardiovascular disease in the 
21st century? Expert Opin Drug Saf. 2014; 13:1227-1239.

doi: $10.1517 / 14740338.2014 .937422$

44. Laufs U, Scharnagl H, Marz W. Statin intolerance. Curr Opin Lipidol. 2015; 26:492501. doi: 10.1097/MOL.0000000000000236

45. Rosenson RS. Trial designs for statin muscle intolerance. Curr Opin Lipidol. 2017; 28:488494. doi: 10.1097/MOL.0000000000000454

46. Bays H. Statin safety: an overview and assessment of the data-2005. Am J Cardiol 2006; 97:6C-26C. DOI: 10.1016/j.amjcard.2005.12.006

47. Backes JM, Ruisinger JF, Gibson CA, Moriarty PM. Statin-associated muscle symptoms-Managing the highly intolerant. J Clin Lipidol. 2017; 11:24-33. doi: 10.1016/j.jacl.2017.01.006

48. Selva-O'Callaghan A, Alvarado-Cardenas M, Pinal-Fendez I, Trallero- Aragu s E, Milisenda JC, Martnez M, et al. Statin-induced myalgia and myositis: an update on pathogenesis and clinical recommendations. Expert Rev Clin Immunol. 2018; 14:215-224. doi: 10.1080/1744666X.2018.1440206

49. Auer J, Sinzinger H, Franklin B, Berent R. Muscle- and skeletal-related side-effects of statins: the tip of the iceberg? Eur J Prev Cardiol. 2016; 23:88-110. doi: $10.1177 / 2047487314550804$

50. Stores ES, Thompson PD, Corsini A, Vladutiu GD, Real FJ, Ray KK, et al. European Atherosclerosis Society Consensus Panel. Statin-associated muscle symptoms: impact on statin therapy-European Atherosclerosis Society Consensus Panel Statement on Assessment, Aetiology, and Management. Eur Heart J. 2015; 36:10121022. doi: 10.1093/eurheartj/ehv043

51. Rosenson RS, Baker SK, Jacobson TA,
Kopecky SL, Parker BA. The National Lipid Association's Muscle Safety Expert Panel. An assessment by the Statin Muscle Safety Task Force: 2014 update. J Clin Lipidol. 2014; 8:S58-S71. doi: 10.1016/j.jacl.2014.03.004

52. Michalska-Kasiczak M, Sahebkar A, Mikhailidis DP, Rysz J, Muntner P, Toth PP, et al. Lipid and Blood Pressure Meta-analysis Collaboration [LBPMC] Group. Analysis of vitamin D levels in patients with and without statin-associated myalgia - a systematic review and meta-analysis of 7 studies with 2420 patients. Int J Cardiol. 2015; 178:111116. doi: $10.1016 / j$.ijcard.2014.10.118

53. Morioka TY, Lee AJ, Bertisch S, Buettner C. Vitamin D status modifies the association between statin use and musculoskeletal pain: a population-based study. Atherosclerosis. 2015; 238:77-82. doi: 10.1016/j.atherosclerosis.2014.11.012

54. Wu Z, Camargo CA Jr, Khaw KT, Waayer D, Lawes CMM, Toop L, et al. Effects of vitamin D supplementation on adherence to and persistence with long-term statin therapy: Downloaded from secondary analysis from the randomized, double-blind, placebocontrolled ViDA study. Atherosclerosis. 2018; 273:59-66. doi: 10.1016/j.atherosclerosis.2018.04.009

55. Catapano AL, Graham I, De Backer G, Wiklund O, Chapman MJ, Drexel $\mathrm{H}$, et al. 2016 ESC/EAS Guidelines for the management of dyslipidaemias. Eur Heart J 2016; 37:2999-3058. https://doi.org/10.1093/eurheartj/ehx180

56. Piepoli MF, Hoes AW, Agewall S, Albus C, Brotons C, Catapano AL, et al. Authors/Task Force Members. 2016 European Guidelines on cardiovascular disease prevention in clinical practice: the Sixth Joint Task Force of the European Society of Cardiology and Other 
Societies on Cardiovascular Disease Prevention in Clinical Practice [constituted by representatives of 10 societies and by invited experts] Developed with the special contribution of the European Association for Cardiovascular Prevention \& Rehabilitation [EACPR]. Eur Heart J 2016; 37:2315-2381. doi: 10.1093/eurheartj/ehw106. Epub 2016 May 23.

57. Pastori D, Polimeni L, Baratta F, Pani A, Del Ben M, Angelico F. The efficacy and safety of statins for the treatment of non-alcoholic fatty liver disease. Dig Liver Dis 2015; 47:4-11. https://doi.org/10.1016/j.dld.2014.07.170.

58. Tsochatzis EA, Bosch J. Statins in cirrhosis - ready for prime time. Hepatology 2017; 66:697-699. https://doi.org/10.1002/hep.29277.

59. Pose E, Trebicka J, Mookerjee RP, Angeli P, Ginès P. Statins: Old drugs a new therapy for liver diseases? Journal of Hepatology 2019; 70:194-202. doi: 10.1016/j.jhep.2018.07.019. Epub 2018 Aug 1

60. Mach F, Ray KK, Wiklund O, Corsini A, Catapano A.L, Bruckert E, et al. Adverse effects of statin therapy: perception vs. the evidence - focus on glucose homeostasis, cognitive, renal and hepatic function, hemorrhagic stroke and cataract. European Heart Journal 2018; 39:2526-2539. doi:10.1093/eurheartj/ehy182

61. Simons M, Keller P, Dichgans J, Schulz JB. Cholesterol and Alzheimer's disease: is there a link? Neurology 2001; 57:1089-1093. DOI: 10.1212/wnl.57.6.1089

62. Farrer LA, Cupples LA, Haines JL, Hyman B, Kukull WA, Mayeux R, et al. Effects of age, sex, and ethnicity on the association between apolipoprotein E genotype and Alzheimer disease. A meta-analysis. APOE and Alzheimer Disease Meta Analysis
Consortium. JAMA 1997; 278:1349-1356.

63. Salat D, Ribosa R, Garcia-Bonilla L, Montaner J. Statin use before and after acute ischemic stroke onset improves neurological outcome. Expert Rev Cardiovasc Ther 2009; 7:1219-1230. doi: 10.1586/erc.09.52.

64. Elias PK, Elias MF, D'Agostino RB, Sullivan LM, Wolf PA. Serum cholesterol and cognitive performance in the Framingham Heart Study. Psychosom Med 2005; 67:2430.

DOI:

10.1097/01.psy.0000151745.67285.c2

65. Mahley RW. Central nervous system lipoproteins: apoE and regulation of cholesterol metabolism. Arterioscler Thromb Vasc Biol 2016; 36:1305-1315. doi: 10.1161/ATVBAHA.116.307023. Epub 2016 May 12

66. Fuentes-Orozco C, Garcia-Salazar SJ, GomezNavarro B, Gonzalez- Espinoza E, ZepedaGonzalez A, Ramrez-Robles JN, et al. Antiinflammatory effect of atorvastatin on the kidney graft of living donor transplants. Ann Transplant. 2018; 23:442-449. doi: 10.12659/AOT.908521

67. Okuyama H, Langsjoen PH, Hamazaki T, Ogushi Y, Hama R, Kobayashi $\mathrm{T}$, et al. Statins stimulate atherosclerosis and heart failure, pharmacological mechanisms. Expert Rev Clin Pharmacol 2015; 8: 189-199. doi: 10.1586/17512433.2015.1011125. Epub 2015 Feb 6.

68. Ravnskov U, Rosch PJ, McCully KS. Statins do not protect against cancer: quite the opposite. J Clin Oncol 2015; 33:810-1. doi: 10.1200/JCO.2014.58.9564. Epub 2015 Jan 20.

69. Cardwell CR, Hicks BM, Hughes C, et al: Statin use after colorectal cancer diagnosis and survival: A population-based cohort 
study. J Clin Oncol 2014; 32:3177- 83. doi: 10.1200/JCO.2013.54.4569. Epub 2014 Aug 4.

70. Ravnskov U, Rosch PJ, McCully KS: The statin-low cholesterol-cancer conundrum. QJM 2012; 105:383-88. https://doi.org/10.1093/qjmed/hcr243

71. Caparros-Martin JA, Lareau RR, Ramsay JP, Peplies J, Reen FJ, Headlam HA, et al. Statin therapy causes gut dysbiosis in mice through a PXR-dependent mechanism. Microbiome. 2017; 5:95. doi: 10.1186/s40168-017-03124

72. Kaddurah-Daouk R, Baillie RA, Zhu H, Zeng ZB, Wiest MM, Nguyen UT, et al. Enteric microbiome metabolites correlate with response to simvastatin treatment. PLoS One 2011; 6:e25482. doi: 10.1371/journal.pone.0025482

73. Muntean DM, Thompson PD, Catapano AL, Stasiolek M, Fabis J, Muntner P, et al. Statinassociated myopathy and the quest for biomarkers: can we effectively predict statinassociated muscle symptoms? Drug Discov Today 2017; 22:85-96. doi: 10.1016/j.drudis.2016.09.001

74. Bellosta S, Corsini A. Statin drug interactions and related adverse reactions: an update. Expert Opin Drug Saf 2018; 17:25-37. doi: $10.1080 / 14740338.2018 .1394455$

75. Fathi HA, Allam A, Elsabahy M, Fetih G, ElBadry M. Nanostructured Lipid Carriers for Improved Oral Delivery and Prolonged Antihyperlipidemic Effect of Simvastatin, Colloids, and Surfaces B: Biointerfaces 2018; 162:236-245 https://doi.org/10.1016/j.colsurfb.2017.11.064

76. Zheng X, Zhang W, Wang Z. Simvastatin preparations promote PDGF-BB secretion to repair LPS-induced endothelial injury through the PDGFR $\beta /$ PI3K/ Akt/IQGAP1 signaling pathway. J Cell Mol Med. 2019; 23:8314-27. DOI: 10.1111/jcmm. 14709 